\title{
Magnetically actuated circular displacement micropump
}

\author{
Markus Gusenbauer $^{1}$. Giulia Mazza ${ }^{1} \cdot$ Thomas Posnicek $^{1} \cdot$ Martin Brandl $^{1} \cdot$ Thomas Schrefl $^{1}$
}

Received: 27 April 2017 / Accepted: 24 November 2017 / Published online: 14 December 2017

(C) The Author(s) 2017. This article is an open access publication

\begin{abstract}
The development process of a magnetically actuated displacement micropump is demonstrated. Two permanent magnets are driven by electromagnets in a circular housing. The magnetic plugs dynamically act as valve or as driving unit. A theoretical model is used to obtain the plug velocities in the system through the calculation of the force equilibria. Especially, the small gap between the channel wall and the plug has a large influence on the resulting pump performance. Final design parameters are obtained by computational fluid dynamics simulations, which predict occurring pressure loads and developing flow rates. Additive manufacturing can be used to build the device. All materials in the fabrication are biocompatible to allow water, liquid foods, and cell-containing fluids like blood to be pumped. A detailed experimental and theoretical comparison is given for two different pump layouts.
\end{abstract}

Keywords Settling velocity $\cdot$ Magnetic bead $\cdot$ CFD $\cdot$ Sphere in tube $\cdot$ Magnetic field $\cdot$ Additive manufacturing

\section{Introduction}

Micropumps are used to pump small amount of fluids through channels in the micrometer regime. The flow rates are set from a fraction to several milliliters per minute. Micropumps can be categorized into displacement and dynamic type pumps [1]. A recent overview of currently available technologies is given by Yokota [2]. Displacement pumps basically have movable parts (e.g., membranes, plugs) that create a flow due to a positive change of displacement. Valves control the direction of the fluid; hence, periodic fluctuations can be typically observed. On the other hand, dynamic pumps use electric or magnetic phenomena to transport fluids directly without using movable parts. Turbines used in pumps are categorized as well to dynamic pumps due to their aperiodic flow.

In this work, we design a displacement micropump by multiscale modeling and demonstrate its functionality by building prototype devices using $3 \mathrm{D}$ printing technologies.

Markus Gusenbauer

markus.gusenbauer@donau-uni.ac.at

1 Center for Integrated Sensor Systems, Danube University Krems, Dr.-Karl-Dorrek-Strasse 30, 3500 Krems, Austria
We use analytical models to describe the motion of magnetic plugs driven by magnetic fields. Based on the placement and current profile, the field-generating electromagnets are derived. Computational fluid dynamics is used for geometrical optimization including the shape and dimensions of the plug with respect to the pipe cross section.

First time introduced in 2001, Hatch et al. [3] proposed a displacement method using ferrofluidics driven by a rotating permanent magnet to pump fluids through a microfluidic regime (Fig. 1 on the left). A fixed permanent magnet plugs ferrofluid to block the direct connection from inlet to outlet. The rotating permanent magnet uses parts of the stationary ferrofluid to create a new rotating plug, which is pushing the fluid. Unfortunately, the possible flow rate is limited due to leakage of the system. In fact, the ferrofluid loses stability, in terms of a closed plug, at too large throughputs. With an increased cross section of the loop, a larger volume needs to be filled with the ferrofluid as well. Pumping is possible as long as the net force from the magnetic field is larger than the pressure load. A promising approach using stiff magnets as a replacement for the ferrofluid was introduced in 2010 [4] and improved by the same group [5]. Compared to previous versions using ferrofluids, considerably high flow rates could be achieved $(13.7 \mathrm{ml} / \mathrm{min}$ with water). Leakage occurs as well and has not been investigated further by this research group. 
Fig. 1 Principle workflow of the pump in accordance with [3] (on the left). Plug A is temporarily fixed, while plug B is pushing the fluid. After one cycle, the plugs swap their positions. Plug $\mathrm{A}$ is the new driving plug and plug B is now temporarily fixed. The arrangement of electromagnets, which create the magnetomotive force for the plugs, is shown on the right
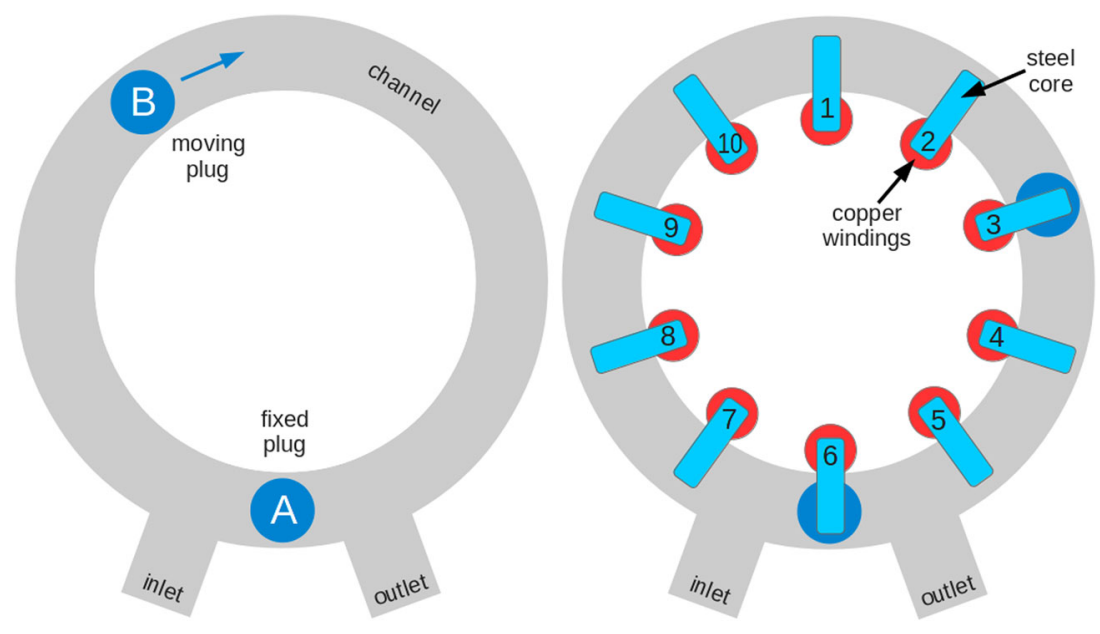

In the fabrication of micropumps, the advantages of additive manufacturing are gaining great popularity (e.g., as shown in healthcare applications [6]). Especially high speed and flexibility are important, not only in the production routines but also as rapid prototyping tool in the development process. Printing very small details in the microscale is of great interest as well in order to build better, faster, smaller, and cheaper devices [7].

\subsection{Content of the paper}

In this work, we will show the development of a pumping device, based on the principle of Hatch et al. [3]. The pump mechanism is shown in Fig. 1, left. A certain leakage of the pumping plug, as mentioned in [4], will be investigated in theory and with computer simulations to understand its influence on the pump performance. The plug is made of a spherical, $\mathrm{NiCu}$-coated $\mathrm{NdFeB}$ permanent magnet in order to achieve a large energy contribution to the force exerted by electromagnets. Based on the calculations, the final design of the pump is printed using the Stratasys J750 3D printer (Stratasys Ltd., USA). Pump performance is measured with pure water and a $35 \%$ glycerin/water $(w / w)$ mixture to mimic the viscosity of blood (Section 4). All materials used in the device are biocompatible to allow water, liquid foods, and cell-containing fluids like blood to be pumped.

\section{Materials and methods}

\subsection{Simulations}

Based on the theory and simulation results in Section 3, we design the pumping device with the open-source Salome platform (salome-platform.org, last visited on 08/11/2016). We use the pre- and post-processing software to establish a three-dimensional geometry of the device. A tetrahedral mesh is generated from the geometries for its use in numerical simulations. The flow inside the pump is simulated using the open-source solver ElmerFEM (www. csc.fi/web/elmer, last visited on 08/11/2016), which is a multiphysics and multi-platform finite element software package. We solve the Navier-Stokes equations in order to obtain steady-state pressure and velocity fields inside the analyzed boundaries. The results are visualized using ParaView (www.paraview.org, last visited on 08/11/2016), which is an open-source, multi-platform data analysis and visualization application. To obtain magnetic field and force information of the air coils and electromagnets, we use a self-written C-code. Here, we use analytical equations to calculate the magnetic field of permanent magnets and ideal solenoids including the interaction with soft and hard magnetic spheres (as done in [8]). The main simulation as well as the final prototype parameters is listed in Table 1.

\subsection{Fast prototyping/additive manufacturing}

Throughout the developing process, we used several 3D printing technologies and materials. Initial designs for simple experiments were made using low-cost 3D printing devices. Consumer printers using fused deposition modeling (material: polylactic acid) or laser sintering (material: polyamid) could not be used, since the proposed pumping design needs a water-resistant material with only little printing tolerance $(<0.1 \mathrm{~mm})$. Stereolithography (material: transparent resin) could be used to print the device, yet commercially printed products (material: transparent resin, i.materialise, Belgium) were finished with a material to prevent UV-radiation, which was water-permeable. Our final prototype is printed with a Stratasys J750 polyjet printer (material: VeroClear, Stratasys Ltd., USA), which allows very precise details of the printed object (Fig. 2). 
Table 1 Simulation and experimental parameters: the range for the Reynolds number $\left(\mathrm{R}_{e}\right)$ is explained by different fluids and different values for tube (channel), sphere (plug), or hollow cone (gap between plug and channel wall) flow

\begin{tabular}{|c|c|c|}
\hline Parameter & Value(s) & Unit \\
\hline \multicolumn{3}{|l|}{ Fluid } \\
\hline Dynamic viscosity $(v)$ & $0.89^{\mathrm{w}}, 3.5^{\mathrm{b}}$ & $\mathrm{mPa} \cdot \mathrm{s}$ \\
\hline Density $(\rho)$ & $1.0^{\mathrm{w}}, 1.06^{\mathrm{b}}$ & $\mathrm{g} / \mathrm{cm}^{3}$ \\
\hline Reynolds number $\left(\mathrm{R}_{e}\right)$ & $10^{2}-10^{4} *$ & \\
\hline \multicolumn{3}{|l|}{ Plug } \\
\hline Sphere diameter & 7 & $\mathrm{~mm}$ \\
\hline \multicolumn{3}{|l|}{ Stainless steel $(1.4034)^{1}$} \\
\hline magn. susceptibility $\chi$ & $\sim 700$ & \\
\hline Saturation flux density $B_{\mathrm{s}}$ & $\sim 1.65$ & $\mathrm{~T}$ \\
\hline \multicolumn{3}{|l|}{$\mathrm{NdFeB} \mathrm{N35^{2 }}$} \\
\hline Remanent flux density $B_{r}$ & $\sim 1.2$ & $\mathrm{~T}$ \\
\hline \multicolumn{3}{|l|}{ Coils } \\
\hline Current I & $1-6$ & A \\
\hline Copper wire diameter & 0.5 & $\mathrm{~mm}$ \\
\hline Windings $\mathrm{N}$ & $100,200,300$ & \\
\hline \multicolumn{3}{|l|}{ Air coil } \\
\hline Diameter | length & $3 \mid 1$ & $\mathrm{~cm}$ \\
\hline \multicolumn{3}{|l|}{$C$-shaped electromagnet } \\
\hline \multicolumn{3}{|c|}{ Core material: ferritic stainless steel } \\
\hline Air gap & 3 & $\mathrm{~cm}$ \\
\hline Core permeability $\mu_{r}$ & $\sim 1000$ & \\
\hline Core length | diameter & $9 \mid 5$ & $\mathrm{~cm}$ \\
\hline \multicolumn{3}{|l|}{ Pump geometry } \\
\hline Diameter | height & $8 \mid 3$ & $\mathrm{~cm}$ \\
\hline \multirow[t]{2}{*}{ Channel diameter } & $7.6^{\mathrm{S}}-11^{\mathrm{S}}$ & $\mathrm{mm}$ \\
\hline & $7.2^{\mathrm{P}}, 7.4^{\mathrm{P}}$ & $\mathrm{mm}$ \\
\hline
\end{tabular}

${ }^{\mathrm{w}}$ water, ${ }^{\mathrm{b}}$ blood, ${ }^{\mathrm{S}}$ simulation only, ${ }^{\mathrm{P}}$ prototype only

${ }^{1}$ Kugel-Winnie, Germany

${ }^{2}$ Powermagnetshop, MTG Europe e.K., Germany
The geometry files for the prints are obtained again from the Salome platform, from which we computed the fluid dynamics.

\subsection{Coil control}

The plugs for the pump are made of spherical NdFeB permanent magnets. In order to achieve high magnetic forces (see Section 3.1 for details), we use ten electromagnets evenly distributed around the circular pump channel (Fig. 1 on the right and Fig. 2). The electromagnetic core is made of steel, which is wounded by a $0.5-\mathrm{mm}$ copper wire. The control unit for the electromagnets (timing, current intensity) is based on an Arduino microcontroller (Arduino MEGA ADK, www.arduino.cc, last visited on 08/11/2016), which is programmed by the open-source Arduino software. The Arduino board has several analog and digital connectors. The electromagnets are controlled by the digital outputs using a pulse width-modulated voltage ( $>4 \mathrm{kHz}$ ). An additional electronic board serves as a driver circuit for the Arduino to control the coil current and the read out of an attached hall sensor (Fig. 3).

Overswinging is prevented by using a ramp function to turn an electromagnet on and off. The design of the electronic board connecting the electromagnets with the Arduino board is created with the commercially available printed circuit board (PCB) software EAGLE (educational license, cadsoft.io, last visited on 08/11/2016) and fabricated using in-house board milling and drilling (ProtoMat S63, LPKF Laser \& Electronics AG, Germany).

\subsection{Experiments}

\subsubsection{Pump performance}

For the pump characterization, a simple circuit with two reservoirs is used to obtain the flow rate vs. pressure load
Fig. 2 Final pump design (parameters are listed in Table 1). To prevent leakiness of the device, slots next to the main channels for sealing rings are implemented. Nonmagnetic plastic screws can be used to adequately connect the two main parts of the device without gluing. Ten electromagnets with a steel core are used to drive the $\mathrm{NdFeB}$ plugs

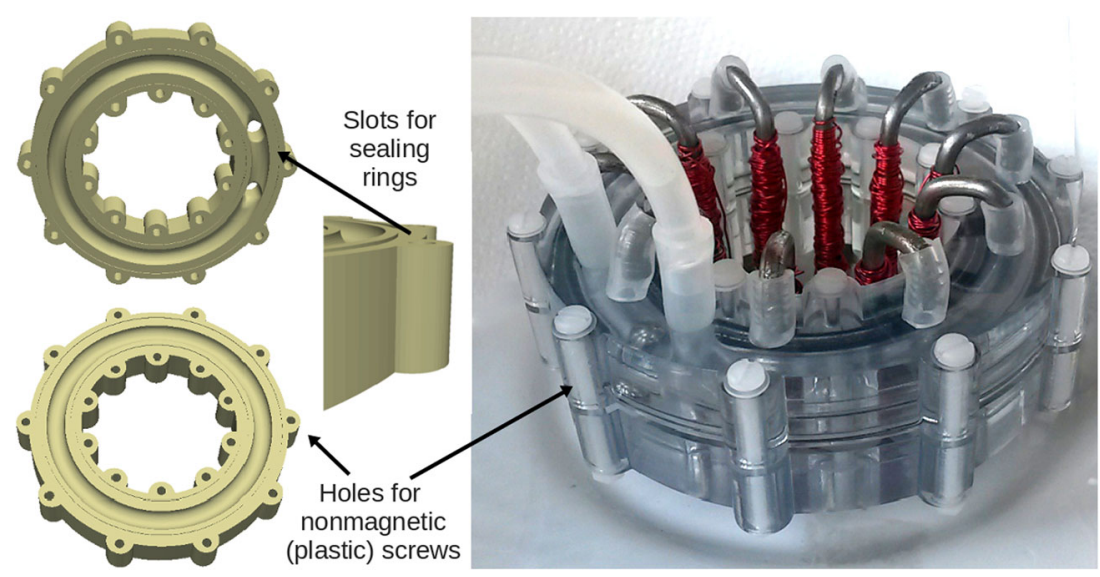




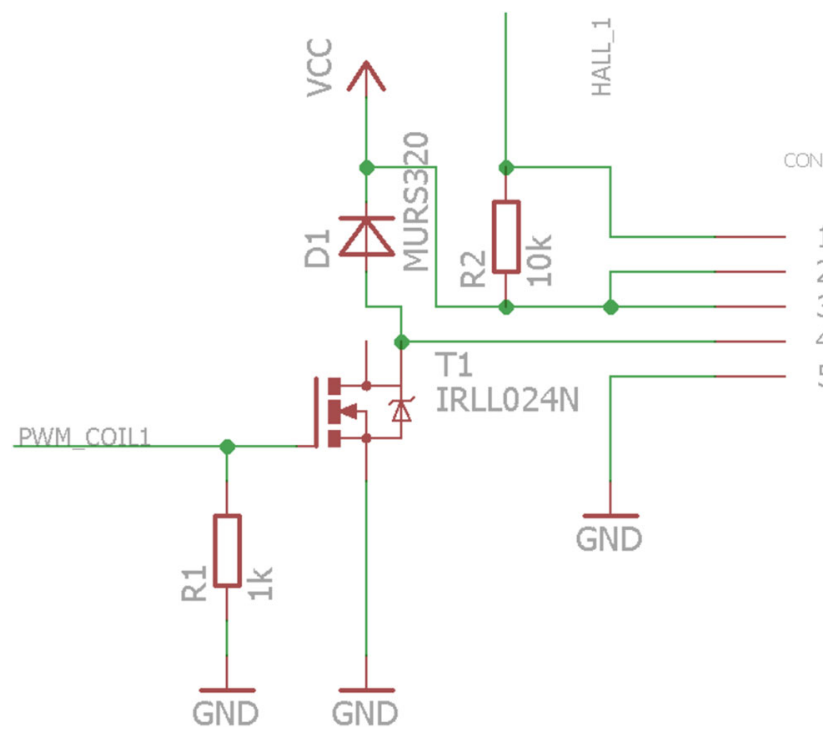

Fig. 3 Electrical circuit diagram of a single electromagnet control. From a digital output of the Arduino microcontroller, a pulse widthmodulated voltage is used to obtain a controlled current between 0 and 6 A (MOSFET T1). The pull-down resistor R1 securely blocks T1, whereas the suppressor diode D1 is used to prevent flyback from the switched inductive load. An additional hall sensor, which uses the pull-up resistor R2, can be connected to get information of the $\mathrm{NdFeB}$ plug position

curves, depending on the pump settings (electromagnet duration time (dt), current $(I)$ ). The curves are obtained for water (viscosity $(v)=1 \mathrm{mPa} \cdot \mathrm{s}$ ) and for a $35 \%$ glycerin/water $(w / w)$ mixture (viscosity $(v)=3 \mathrm{mPa} \cdot \mathrm{s}$ at room temperature). The flow rate is measured with a flow meter positioned between the pump outlet and the outlet reservoir (HT110 with sensor H4XL, Transonic Systems Inc., Ithaca, NY, USA), while the afterload is given by the height difference between the reservoirs.

The electromagnets are anti-cyclically activated to obtain a pumping mechanism. In Fig. 1 on the right, the arrangement of electromagnets with their IDs is shown. The pumping cycle includes an active and a neutral period. The spherical plugs start the cycle in positions 3 and 6. During the neutral period, the electromagnets 3 to 6 , as well as 6 to 9 , are activated sequentially to bring plug B in position 6 and plug A in position 9. After plug B has reached position 6 , it is kept fixed to work as a valve between the shortest connection from inlet to outlet. Now, the active period of the cycle is effectively delivering a flow rate. During this part, the electromagnets 9 to 3 are sequentially activated to drive plug A and pump the fluid. When plug A reaches position 3 , the cycle starts again.

\subsubsection{Cytotoxicity tests}

The 3D printing material (VeroClear, Stratasys Ltd., USA) is a proprietary UV-curable poly(methyl metha-crylate)based material which has already been used for cell culture applications $[9,10]$; therefore, its biocompatibility is not further investigated. The tubing material (Tygon ${ }^{\circledR}$ 3350, Saint-Gobain, France) is a special silicone for pharmaceutical and medical applications with documented biocompatibility. When no data about the biocompatibility of the materials can be found in literature, cytotoxicity tests are performed on erythrocytes, in accordance with the norm ISO 10993-5 on "Tests for in vitro cytotoxicity." The tested material is incubated in RPMI cell culture medium for $24 \mathrm{~h}$ at $37^{\circ} \mathrm{C}$. After incubation, the material samples are removed and erythrocytes are added. The viability of the cells is measured after $24 \mathrm{~h}$ with the CellTiter-Blue ${ }^{\circledR}$ test (Promega, Madison, WI, USA).

\section{Theory and calculations}

\subsection{Plug control}

The force on the magnetic plug $\left(\mathbf{F}_{\text {plug }}\right)$ can be written as the external magnetic gradient force $\left(\mathbf{F}_{\mathrm{g}}\right)$, reduced by the drag force $\left(\mathbf{F}_{\mathrm{d} \text {,tube }}\right)$ of the fluid on the plug in a narrow tubing (incorporating the hydrodynamics close to the channel wall) and the friction of the channel wall $\left(\mathbf{F}_{\mathrm{r}}\right)$ (in case of contact with the plug).

$\mathbf{F}_{\text {plug }}=\mathbf{F}_{\mathrm{g}}-\mathbf{F}_{\text {d,tube }}\left(-\mathbf{F}_{\mathrm{r}}\right)$

The gradient force $\left(\mathbf{F}_{\mathrm{g}}\right)$ on a magnetic material is given by the negative gradient of the energy of the magnetic dipole moment $\mathbf{m}$ in the field $\mathbf{B}$.

$\mathbf{F}_{\mathrm{g}}=-\nabla(E)=\nabla(\mathbf{m} \times \mathbf{B})$

During laminar flow, the Stokes Law is used to calculate the drag force $\left(\mathbf{F}_{\mathrm{d}}\right)$ on an object in a fluid with the drag coefficient $(\beta)$ and the relative velocity $(\mathbf{v})$ of the particle. $\beta$ contains the particle radius $(r)$ and the dynamic viscosity $(v)$ of the fluid. Our proposed device works in the intermediate Reynolds number regime between laminar and turbulent flows $\left(\mathrm{R}_{e} \approx 10^{2}-10^{4}\right)$. In case of unbounded flow (no channel walls), we use the term $\mathbf{F}_{\mathrm{d} \text {,inf. }}$.

$\mathbf{F}_{\mathrm{d}, \text { inf }}=\beta \mathbf{v}_{\text {inf }}=6 \pi v r \mathbf{v}_{\text {inf }}$

Based on Francis [11], the empirical equation for the relation between the settling velocity of a falling sphere in an unbounded fluid $\left(\mathbf{v}_{\mathrm{s}, \text { inf }}\right)$ and the sphere settling velocity in a tube $\left(\mathbf{v}_{\mathrm{s}, \text { tube }}\right)$ can be written as

$\mathbf{v}_{\mathrm{s}, \text { tube }}=\mathbf{v}_{\mathrm{s}, \text { inf }} K=\mathbf{v}_{\mathrm{s}, \text { inf }}\left(\frac{1-d / D}{1-0.475 d / D}\right)^{4}$

with the tube diameter $(D)$ and sphere diameter $(d)$. The settling velocity $\left(\mathbf{v}_{\mathrm{s}}\right)$ is reached in the force equilibrium of the fluidic drag force, the gravitational force, and the buoyancy force. The dynamics, right before the sphere 
reaches the settling velocity, are not described. We assume that the scaling coefficient $(K)$ of bounded to unbounded flow is constant during acceleration of the sphere (in non equilibrium state). There are many follow-up publications on the settling velocity for various flow regimes [12]; nevertheless, we are focusing on Francis work (Eq. 4) for the theoretical model of the plug.

\subsubsection{Electromagnets vs. air coils}

The magnetic flux density $\left(B_{0}\right)$ in the center of an air coil is calculated by the permeability of vacuum $\left(\mu_{0}\right)$ times the magnetomotive force (wire turns per coil length $n=N / L$ and current $(I)$ ).

$B_{0}=\mu_{0} n I$

Using a ferromagnetic core, $B_{0}$ is increasing by the relative permeability $\left(\mu_{r}\right)$ of the core material to

$B_{0}=\mu_{r} \mu_{0} n I$

In Fig. 4, we show the comparison of magnetic field lines originated by an air coil and electromagnet $(N=200, I=$ $1 \mathrm{~A})$. Magnetic field lines are much more focused in the air gap of electromagnets.

Due to limited space and the circular shape, it is easier to mount electromagnets to the proposed pumping device. In case of a $\mathrm{C}$-shaped electromagnet with an air gap with distance $(d)$ and core length $(l)$, the magnetic flux density between the poles is calculated by

$B_{0}=\frac{\mu_{r} \mu_{0} N I}{l+d\left(\mu_{r}-1\right)}$.

With $\mu_{r}>>l$, the field reduces to

$B_{0}=\frac{\mu_{0} N I}{d}$.

As in the work of Gusenbauer et al. [13], we compute the magnetic gradient force $\left(\mathbf{F}_{\mathrm{g}}\right)$ using the analytical equations of an ideal solenoid [14]. In order to model the electromagnet, we assume to have two identical solenoids with the same length and material as the electromagnet. The electromagnetic core is made of ferritic stainless steel, which provides a magnification of the magnetic field by about 1000 ( $\mu_{r}$ in Eq. 6). In Fig. 5, we compare the magnetic gradient force on a spherical plug applied from an air coil $\left(F_{\mathrm{g}, \text { air }}\right)$ and electromagnet $\left(F_{\mathrm{g}, \mathrm{em}}\right)$ with different number of wire turns $(N)$.

The positive slope of the force field pulls the spherical plug into the center of the coil/electromagnet $(z=0)$. Right after the central position, the direction of the force changes and the plug is pushed back. Therefore, without turning off or changing the direction of the current, the plug is trapped. Using C-shaped electromagnets with an air gap of $3 \mathrm{~cm}$, the force on a spherical plug is $f$ times larger than the force created by an air coil with the same number of wire turns $(N)$ and current $(I)$. The factor $(f)$ depends on the material of the plug and is around 7 for a $\mathrm{NdFeB}$ plug, and 47 for a steel plug. In this comparison, the air gap of the electromagnet equals the diameter of the air coil. This minimum distance is necessary due to the dimensions of the 3D-printed pumping case.

\subsubsection{Hard vs. soft magnetic plug}

At room temperature, conventional $\mathrm{NdFeB}$ permanent magnets have a constant magnetization. They are the strongest commercially available magnets with a large energy contribution $(E)$ on the magnetic gradient force $\left(\mathbf{F}_{\mathrm{g}}\right)$ (Eq. 2). Soft magnetic plugs require a stronger magnetic field induced by the external magnetic source for the same energy contribution. At equal magnetomotive force $(N I)$, the magnetic gradient force acting on a spherical $\mathrm{NdFeB}$ plug is about $5 \times 10^{3}$ (electromagnet, $N I=300$ ) up to $9 \times 10^{4}$ (air coil, $N I=100$ ) times larger than on a spherical steel particle (Fig. 5). In order to drive the plugs in the device with limited space, we choose to use hard magnetic $\mathrm{NdFeB}$ particles and electromagnets. Downside of

Fig. 4 Magnetic field lines of an air coil (on the left) and C-shaped electromagnet (on the right) with 200 turns and applied current of $1 \mathrm{~A}$. Diameter of air coil and electromagnets air gap size are the same. The spherical magnetic plug is moving from left to right
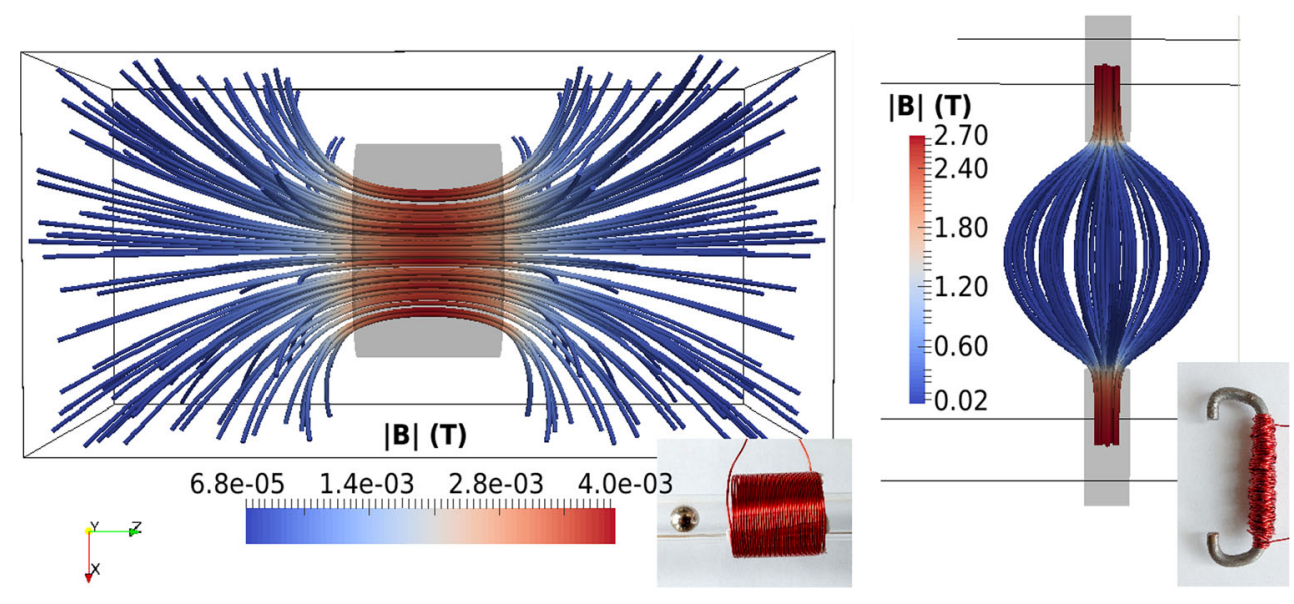
Fig. 5 Magnetic gradient force $\left(\mathbf{F}_{\mathrm{g}}\right)$ of an electromagnet on a spherical magnetic plug in function of the number of wire turns $N(I=1 \mathrm{~A})$. A hard magnetic NdFeB sphere (left axis) and a soft magnetic steel particle (right axis) are compared. The air gap of the $\mathrm{C}$-shaped electromagnet is equal to the diameter of the air coil (see Fig. 4). The shapes of the two NdFeB sphere curves are about the same (likewise for the two steel sphere curves). They differ only in their amplitudes with a factor $(f)$

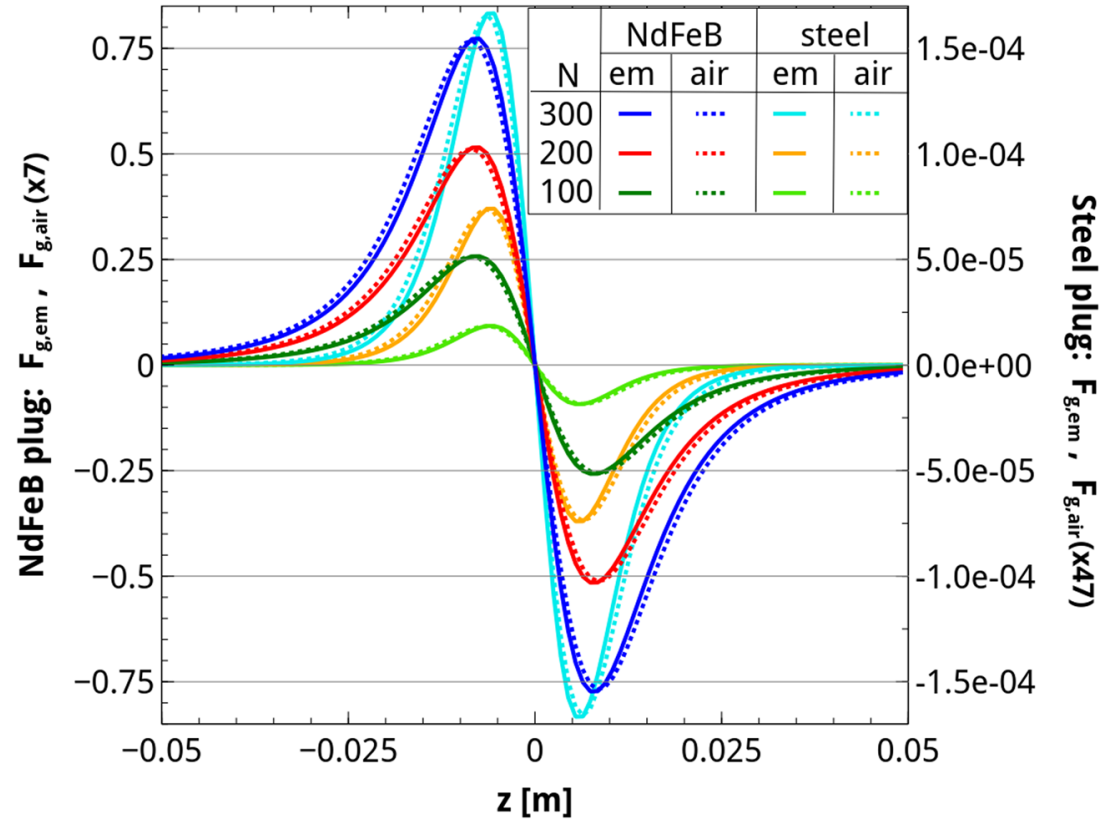

the use of hard magnetic plugs is their possible involuntary aggregation. In fact, the aggregation of soft magnetic plugs can be easily reversed by turning off the field, while in order to split two $\mathrm{NdFeB}$ particles, the pump needs to be opened.

\subsection{Pump performance}

\subsubsection{Plug velocity—ideal pumping cycle}

To obtain the final velocity of a plug $\left(\mathbf{v}_{\text {plug }}\right)$ inside the pumping device, we need to adapt the equation of motion of the plug (Eq. 1).

Right now, the wall scaling coefficient $(K)$ in Eq. 4 is set such, that with narrow tube $K \rightarrow 0$ and within infinite flow $K \rightarrow 1$. In the model, we require $\mathbf{v} \rightarrow 0$ at narrow tubing and $\mathbf{v} \rightarrow \mathbf{v}_{\text {inf }}$ otherwise.

The equation of motion in infinite flow is given as

$m \mathbf{a}=\mathbf{F}_{\mathrm{g}}-\mathbf{F}_{\mathrm{d}, \text { inf }}=\mathbf{F}_{\mathrm{g}}-\beta \mathbf{v}_{\mathrm{inf}}$

For a constant plug flow without acceleration the solution of the unbounded plug velocity $\mathbf{v}_{\text {inf }}$ is

$\mathbf{v}_{\text {inf }}=\mathbf{F}_{\mathrm{g}} / \beta$

Now, two possibilities can be considered to take into account the tube interaction with the plug. The first is to add an additional force term $\left(\mathbf{F}_{\mathrm{w}}\right)$ to Eq. 9. The second is to directly modify the drag coefficient in Eq. 3. In the following passages, we show both scenarios and compare the results. In the calculations, we include neither the possible friction force $\left(\mathbf{F}_{\mathbf{r}}\right)$ of plug and channel wall, nor rotations of the plug, although they could be observed in experiments. The data for the magnetic gradient force
$\left(\mathbf{F}_{\mathrm{g}}\right)$ are taken from the solenoid force calculation (Fig. 5). For simplicity, only the positive force slopes of the electromagnets, connected in serial, have been taken into account (several pumping cycles as shown in Fig. 6). In the experiments, this case would require a perfect timing of the electromagnets, which is unrealistic, unless using complex equipment for sensing and actuation.

\section{Additional force term $\left(F_{\mathrm{w}}\right)$}

Adding a negative force term $\left(F_{\mathrm{w}}\right)$ for the tube interaction to Eq. 9 gives us

$m \ddot{z}=F_{\mathrm{g}}-\beta \dot{z}-F_{\mathrm{w}}$

We now want to compute $F_{\mathrm{w}}$. In equilibrium, the left-hand side of Eq. 11 is zero and $\dot{z}$ in Eq. 11 becomes the terminal

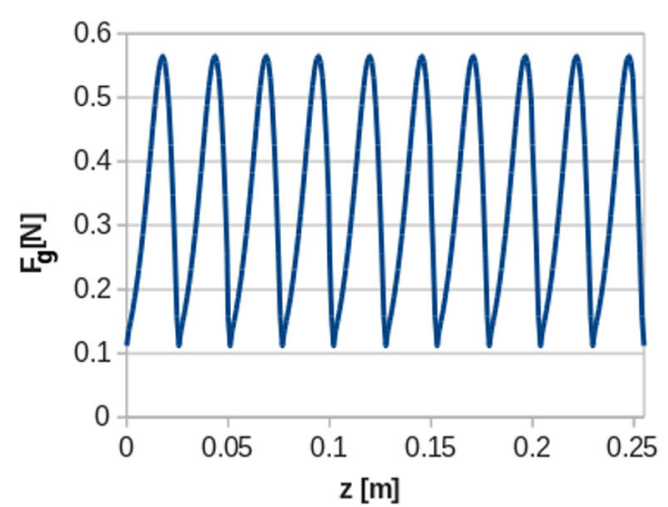

Fig. 6 Magnetic gradient force $\left(\mathbf{F}_{\mathrm{g}}(z)\right)$ of an idealized pumping cycle. Only the positive force slopes of electromagnets with 200 turns and applied $1 \mathrm{~A}$ are placed in serial 
Fig. 7 The plug velocities and positions (NdFeB) are shown for a setup with electromagnets and air coils $(N=200, I=1 \mathrm{~A})$ in water and blood. The gap between plug and channel wall is set from $0.1 \mathrm{~mm}$ to $0.5 \mathrm{~mm}$. The solutions are obtained by Eq. 13 using an additional force term $F_{\mathrm{w}}$ (on the left) and Eq. 16 using a modified drag coefficient $\beta^{*}$ (on the right)
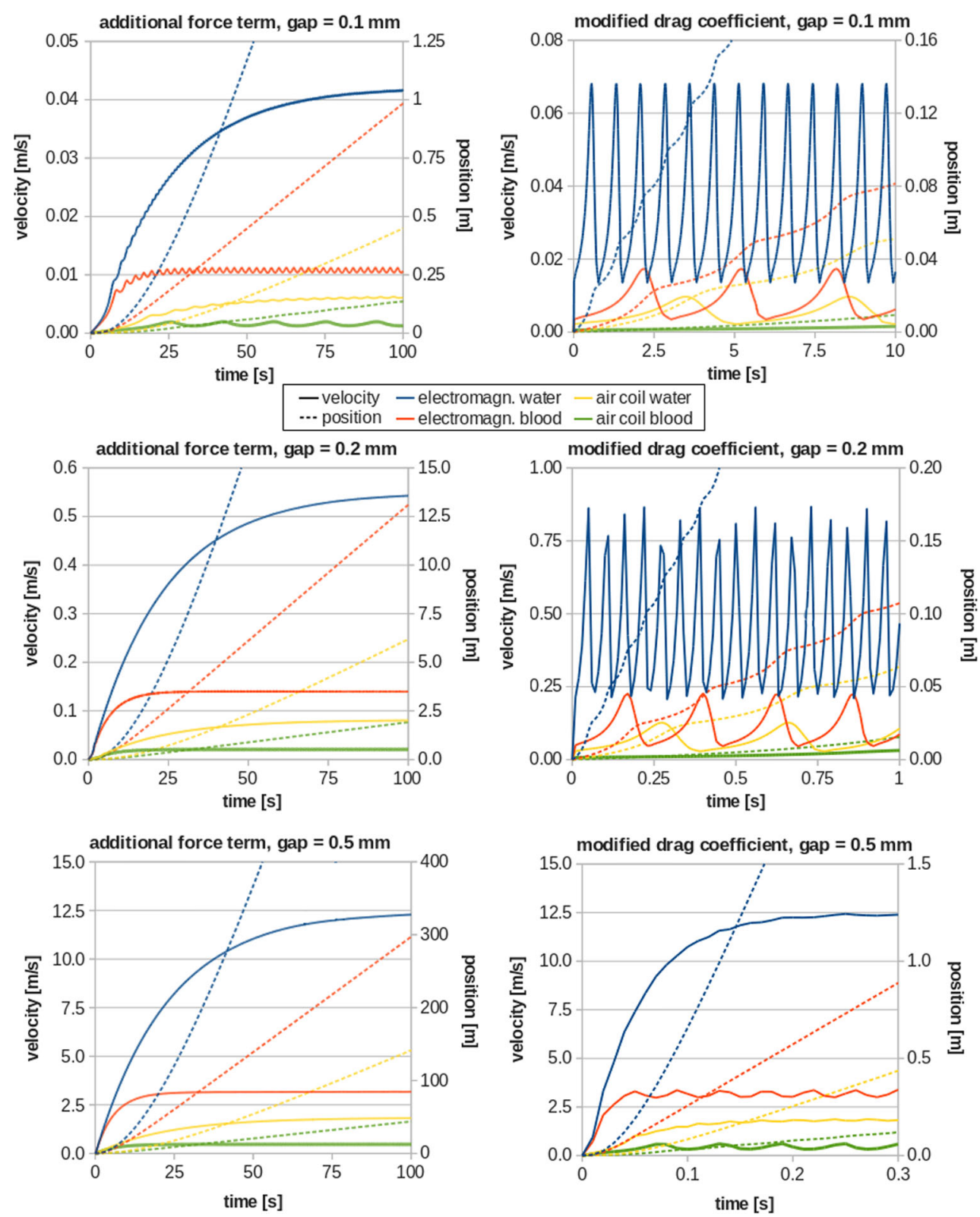

velocity $\dot{z}=v_{\mathrm{s} \text {,tube }}=\left(F_{\mathrm{g}} / \beta\right) K$ by using Eqs. 4 and 10 . This equation gives

$F_{\mathrm{w}}=F_{\mathrm{g}}(1-K)$,

resulting in the final equation of motion for the plug in a tube with an additional force term

$m \ddot{z}=F_{\mathrm{g}}(z) K-\beta \dot{z}$.

Other than in Gusenbauer et al. [15], where we obtain the final particle position at force equilibrium of magnetic gradient force $\left(F_{\mathrm{g}}\right)$ and the fluidic drag force $\left(F_{\mathrm{d}}\right)$, the plug velocity $(\dot{z})$ is derived by the force equilibrium of the adapted magnetic gradient force $\left(F_{\mathrm{g}} K\right)$ and $F_{\mathrm{d}}$. Our new equation limits the magnetic gradient force according to the tube dimensions ( $F_{\mathrm{g}} K \rightarrow 0$ at narrow tubing).
In order to show the velocity of a $\mathrm{NdFeB}$ spherical plug for various scenarios, we compare electromagnets and air coils with 200 turns and an applied current of $1 \mathrm{~A}$ each (Fig. 7 on the left). The fluidic properties are set for water and blood (see Table 1). Small oscillations can be observed, which is caused by the magnetic gradient force $\left(F_{\mathrm{g}}(z)\right)$ input. Until the plugs get to their final velocity in force equilibrium, it takes tens of seconds.

\section{Modified drag coefficient $\left(\beta^{*}\right)$}

In the previous scenario, we added an extra force term $\left(F_{\mathrm{w}}\right)$ to the equation of motion. Here, we change the fluidic drag force directly by a modified drag coefficient $\left(\beta^{*}\right)$.

$m \ddot{z}=F_{\mathrm{g}}-\beta^{*} \dot{z}$ 


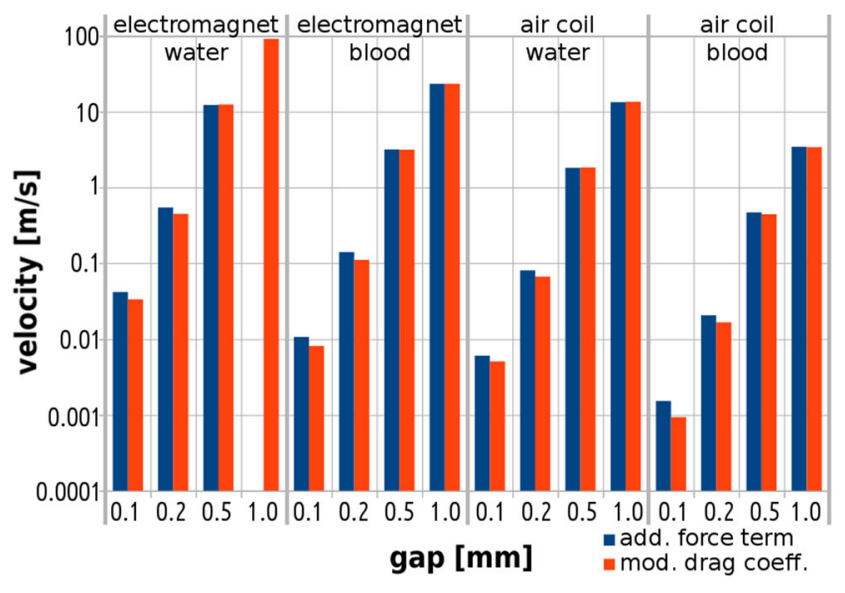

Fig. 8 The final settling velocities are compared for the equations of motion with an additional force term $\left(\mathbf{F}_{\mathrm{w}}\right)$ and with a modified drag coefficient $\left(\beta^{*}\right)$. The missing bar is caused by instabilities in the calculation, especially with large gaps undesired velocity peaks occur

With no acceleration and inserting again Eqs. 4 and 10, it gives us a new drag coefficient

$\beta^{*}=\beta / K$

The new equation of motion for the plug in the tube with a modified drag coefficient is given as

$m \ddot{z}=F_{\mathrm{g}}(z)-\beta \dot{z} / K$.

The plug velocities and positions (NdFeB) resulting from Eq. 16 are shown in Fig. 7 on the right for various gaps between plug and channel wall using electromagnets and air coils, for water and blood.

Both Eqs. 13 and 16 lead to the same terminal velocity $\left(v_{\mathrm{s}, \text { tube }}\right)$ in agreement with the work of Francis [11] whereby gravity is replaced by the magnetic gradient field $\left(F_{\mathrm{g}}\right)$. In Eq. 13, the force acting on the particle is smaller, whereas in Eq. 16, the force is the full magnetic force but the

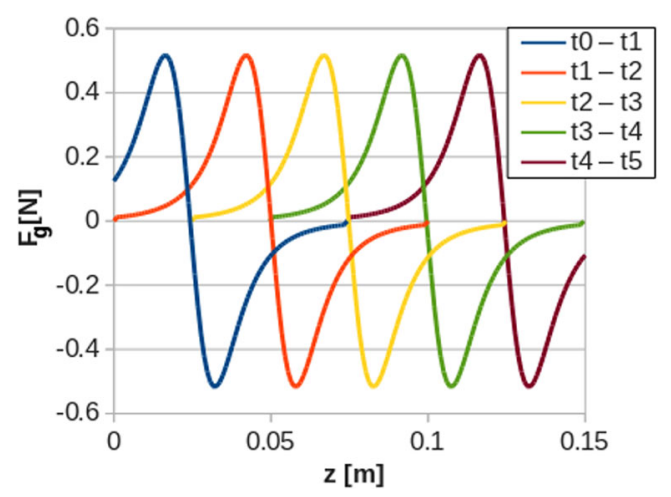

Fig. 9 The real pumping cycle is set in time periods (dt) in which only one single electromagnet $(N=200, I=1 \mathrm{~A})$ is activated $\mathbf{F}_{\mathrm{g}}(z, t)$

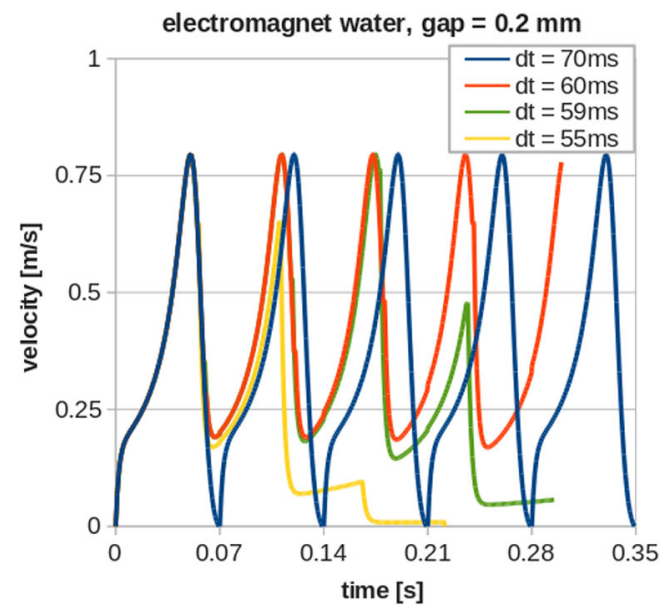

Fig. 10 The response of the NdFeB plug in water with a gap of $0.2 \mathrm{~mm}$ on the time- and space-dependent magnetic gradient force $\left(\mathbf{F}_{\mathrm{g}}(z, t)\right)$ is shown for different duration times (dt) of five electromagnets with 200 turns and applied $1 \mathrm{~A}$ (half pumping cycle). The equation of motion with the modified drag coefficient $\left(\beta^{*}\right)$ is used

damping is larger. Therefore, there is a noticeable difference in acceleration of the plugs compared to the additional force term $\left(F_{\mathrm{w}}\right)$ in the previous section. In Eq. 16, the final velocities in force equilibrium are reached within a few seconds, other than tens of seconds (Eq. 13). Oscillations are much more dominant in the solution of small gaps as well. In experiments, the settling velocity $\left(v_{\mathrm{s}}\right)$ is reached within a short time span [16]. In our pumping system, the terminal velocity is reached in fractions of seconds.

The final settling velocities for the two approaches in equilibrium force are very similar (additional force term $\left(F_{\mathrm{w}}\right)$ and modified drag coefficient $\left(\beta^{*}\right)$ in Fig. 8). Only with small gaps, the values with the modified drag coefficient $\left(\beta^{*}\right)$ are slightly smaller. For oscillating velocities in Fig. 7, an average value is taken.

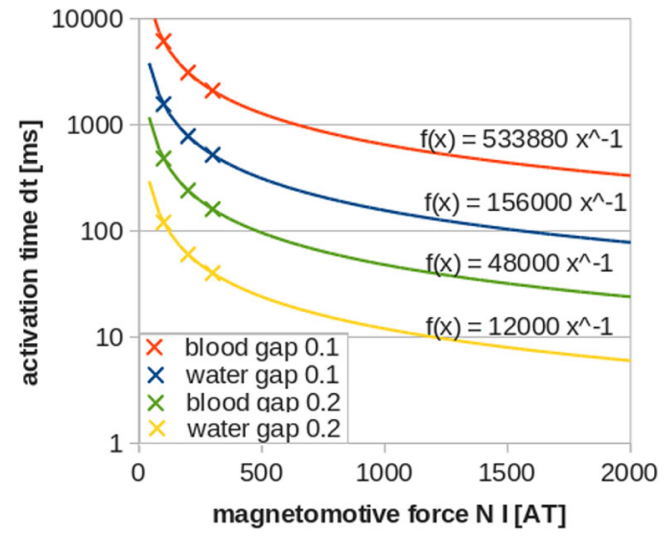

Fig. 11 Optimal duration times (dt) are shown for blood and water with gaps of 0.1 and $0.2 \mathrm{~mm}$. Reciprocal fitting curves are used to estimate the optimal duration times for a given magnetomotive force $(N I)$ in Ampere-turns (AT) 


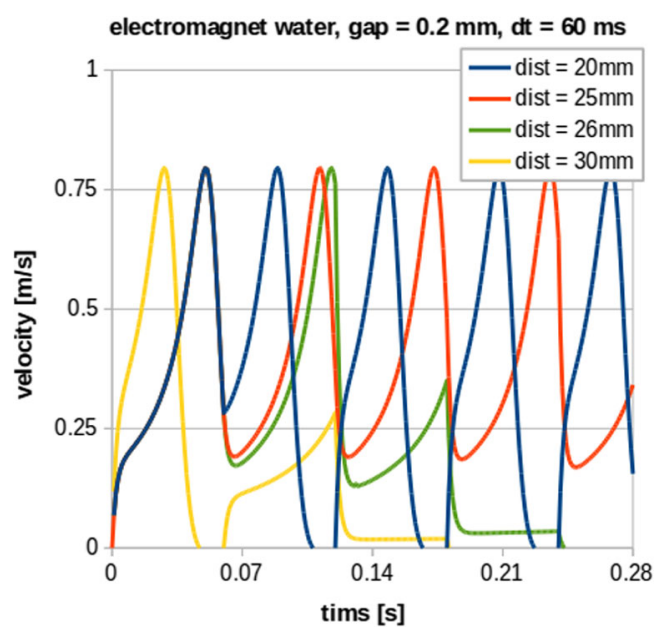

Fig. 12 Similar to Fig. 10, the response of the NdFeB plug in water with a gap of $0.2 \mathrm{~mm}$ on the time- and space-dependent magnetic gradient force $\left(\mathbf{F}_{\mathrm{g}}(z, t)\right)$ is shown for a fixed duration time $(\mathrm{dt})$ of $60 \mathrm{~ms}$ (electromagnets with 200 turns and applied $1 \mathrm{~A}$ ). The distance between the electromagnet is varied from 20 to $30 \mathrm{~mm}$. The equation of motion with the modified drag coefficient $\left(\beta^{*}\right)$ is used

\subsubsection{Plug velocity—real pumping cycle}

In the previous section, we obtained the final plug velocity by an idealized magnetic gradient force $\left(\mathbf{F}_{\mathrm{g}}\right)$. Only the positive force slopes of Fig. 6 are used. In experiments, we cannot avoid the negative force terms unless using expensive equipment. The pump uses ten electromagnets connected in serial to drive the spherical $\mathrm{NdFeB}$ plug. An Arduino microcontroller set timing and current of the electromagnets (as explained in Section 2.3). The electromagnets are sequentially activated with a duration time (dt). In order to calculate the plug velocities and positions for the real pumping cycle, we solve the equation of motion with the time- and space-dependent magnetic gradient force $\left(\mathbf{F}_{\mathrm{g}}(z, t)\right)$ (Fig. 9).
In experiments, we can observe a very fast response of the $\mathrm{NdFeB}$ plug on the magnetic gradient force $\left(\mathbf{F}_{\mathrm{g}}\right)$. Therefore, we are solving Eq. 16 with the modified drag coefficient $\left(\beta^{*}\right)$. Figure 10 shows different duration times (dt) of electromagnetic driven $\mathrm{NdFeB}$ plugs $(N=200$, $I=1 \mathrm{~A})$ in water.

The values of $\mathrm{dt}$ must be chosen carefully. If set too long, the plug is slowed down to total standstill $(\mathrm{dt}=70 \mathrm{~ms}$ ). Some optimum in speed can be achieved with $\mathrm{dt}=60 \mathrm{~ms}$, where a steady flow can be achieved. Reducing dt below $59 \mathrm{~ms}$ has the effect, that the plug misses the next force information and it stops. It happens earlier if reducing $\mathrm{dt}$ even more $(\mathrm{dt}=55 \mathrm{~ms})$. Changing water to blood for the very same setup increases the optimal duration time to about $240 \mathrm{~ms}$.

The optimal duration time (dt) depends on the magnetomotive force $N I$, the viscosity of the fluid and the gap between the plug and the channel walls. Figure 11 shows the smallest duration times that lead to stable forward motion for a given magnetomotive force. We approximate the optimal duration time as function of the magnetomotive force by a reciprocal fit $(f(x))$.

\subsubsection{Density of electromagnets}

The design of the circular pump is a limiting factor for placing the electromagnets to certain channel positions. The height of the pumping case defines the minimum air gap of the C-shaped electromagnets. Mounting slots for the connection of the two half cases occupy space as well (Fig. 2). Nevertheless, the electromagnets can be placed inside or outside the main channel loop. The distance between the electromagnets in the previous analysis is set to $25 \mathrm{~mm}$. In Fig. 12, we shift the distance between the electromagnets (200 turns, $1 \mathrm{~A}$ ) from 20 to $30 \mathrm{~mm}$ with a fixed duration time (dt) of $60 \mathrm{~ms}$. The functionality of the pump is very sensitive on the given distance between the electromagnets. Increasing the distance by just $1 \mathrm{~mm}$ leads

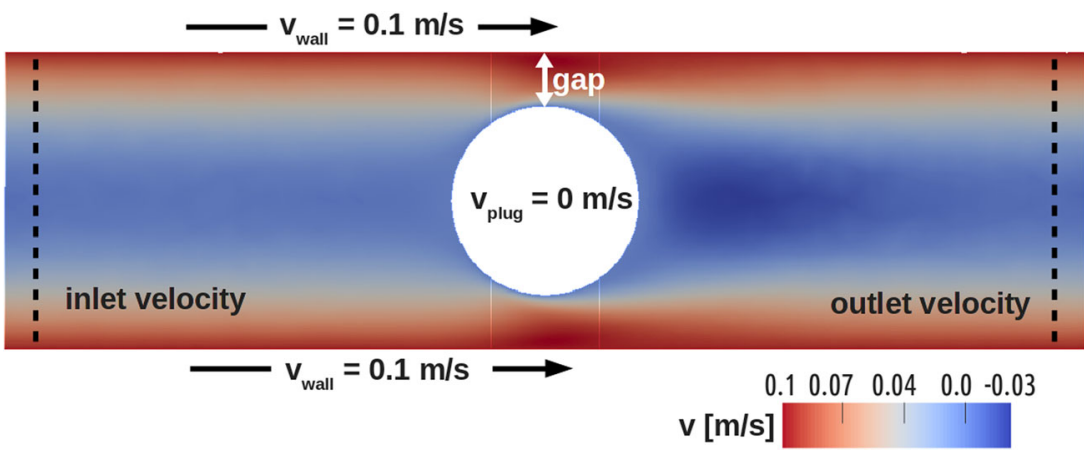

Fig. 13 The three-dimensional simulation setup consists of a fixed plug (diameter of $7 \mathrm{~mm}$ ) and a moving cylindrical boundary (length of $4 \mathrm{~cm}$, diameter from 7.6 to $11 \mathrm{~mm}$ ) to mimic the moving plug in the channel (similar to [17]). The diameter of the channel is varied to compare different gaps between plug and channel wall. The simulated tube is cut longitudinally to visualize and analyze the data 
to a total standstill of the plug. Here, the plug misses the next attracting electromagnet. Choosing a distance too close to each other leads to small standstills as well because of the negative gradient force $\left(\mathbf{F}_{\mathrm{g}}\right)$ after the electromagnets axis, which hinders the plug from moving forward. In this work, we are using a fixed amount of ten electromagnets due to the chosen design. The number can be altered according to a different design; consequently, the magnetomotive force of the electromagnets must be changed carefully.

\subsubsection{Flow rate}

The pumping efficiency is related to the leakage that occurs between the plug and the channel wall (Fig. 1). No gap would be advantageous for the pump performance, but, due to material discontinuities and wall friction, it cannot be avoided. In order to analyze the influence of the gap in terms of pump performance, we run a steady-state simulation of a fixed plug and a moving channel wall with Elmer in three dimensions (Fig. 13). The moving boundary shall imitate the movement of the plug (similar to [17]).

In the simulations, some limitations are encountered when dealing with very small gaps. In fact, gaps below $0.3 \mathrm{~mm}$ drastically increase the memory usage of the computer, due to a very dense tetrahedral mesh close to the gap. Despite of an adaptive mesh size (Fig. 14), simulations with very small gaps cannot be performed. We are using at least ten mesh layers to fill the smallest spatial space in the geometry. The overall mesh for a gap of $0.3 \mathrm{~mm}$ has about 2.5 Mio elements.

In the simulations, we vary the gap between the plug and the channel wall. In Fig. 15, we show the fluid velocities at the inlet of the channel. The velocities occurring at the outlet are approximately the same. The simulations

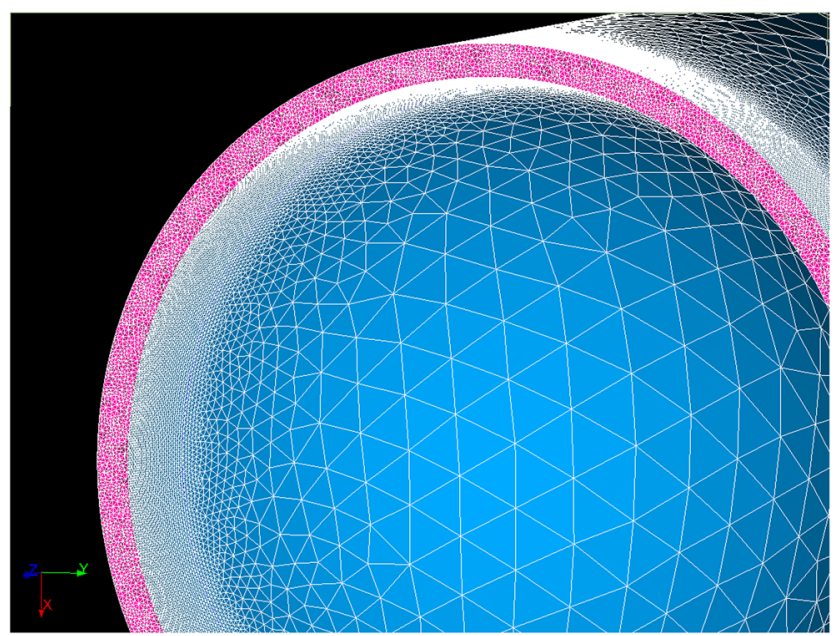

Fig. 14 The channel cross section is shown with a gap size of $0.3 \mathrm{~mm}$ from plug to channel wall. An adaptive mesh is used to apply at least ten mesh layers in the smallest spatial dimension

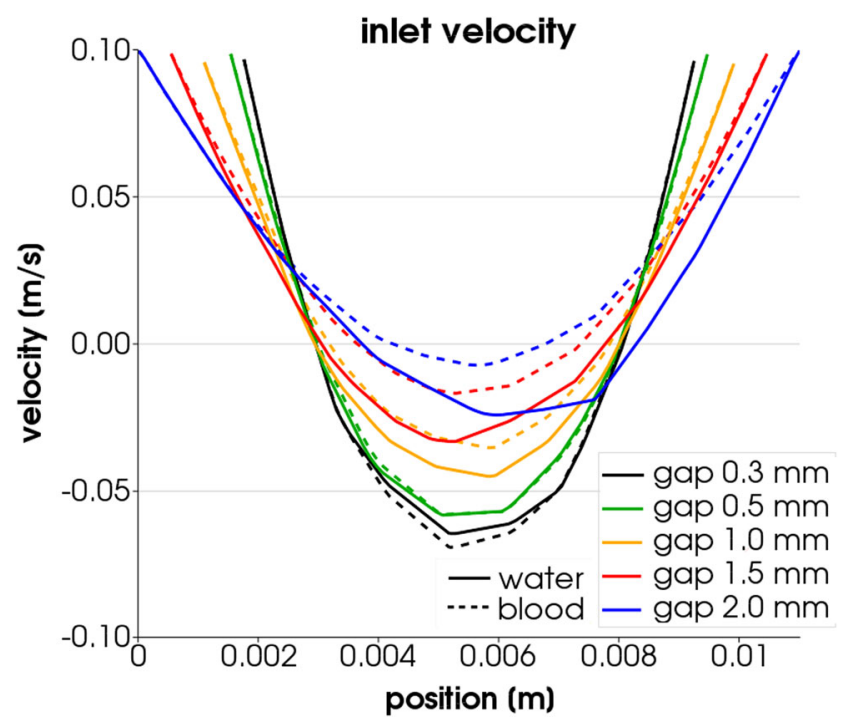

Fig. 15 The fluid velocity for varying gaps is compared using threedimensional Navier-Stokes simulations in Elmer. The data for the plots are obtained at the inlet velocity line (Fig. 13). The velocities are shown as a function of position across the tube

are computed in the intermediate Reynolds number regime between laminar and turbulent flows $\left(\mathrm{R}_{e} \approx 10^{2}-10^{4}\right)$. Consequently, the resulting velocity profiles show small fluctuations.

From the parabolic velocity profile at the channel inlet (Fig. 15), we obtain the mean velocity $\left(v_{\text {mean }}=v_{\max } / 2\right)$ for calculating the flow rate $\left(Q=v_{\text {mean }} A\right)$. We run simulations with a moving channel wall and a fixed plug (Fig. 13); therefore, $v_{\max }$ is given by $v_{\text {wall }}-v_{\text {min }}$. In Fig. 16, the developing flow rates are shown for water and blood. Due to an increasing cross section $A$ of the channel with larger

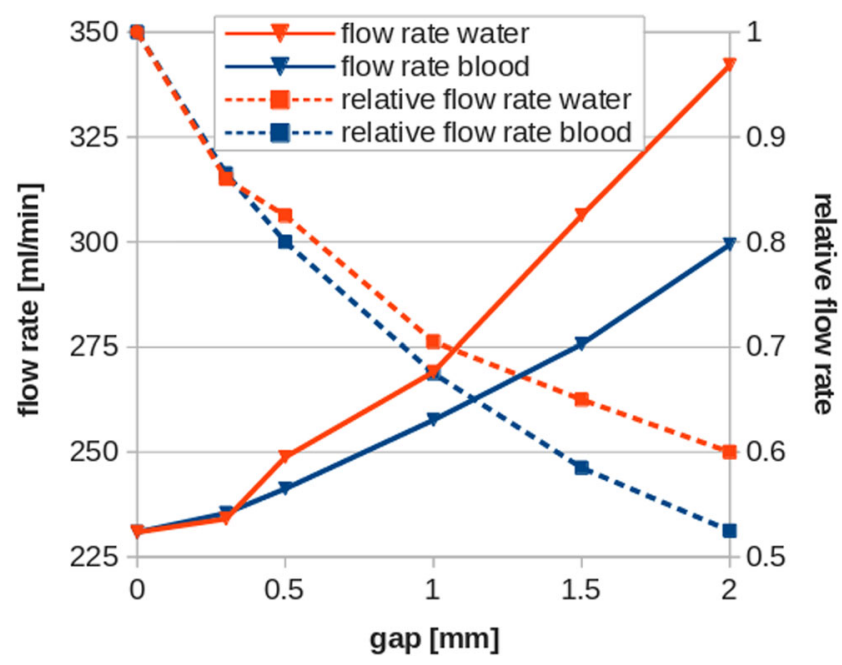

Fig. 16 The flow rate for different gaps is calculated using the mean velocities from the parabolic flow profiles (Fig. 15). The relative flow rate $\left(Q / Q_{\text {ideal }}\right)$ is shown on the right axis. The ideal flow rate $Q_{\text {ideal }}$ is the flow rate, that is achieved without a gap 


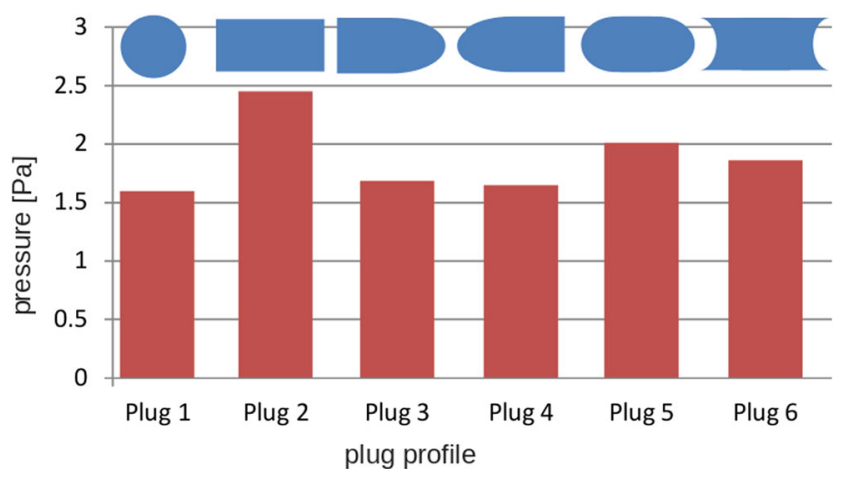

Fig. 17 The plug geometry has an influence on the developing pressure during pumping. With a rectangular plug profile, the highest pressure is induced with a constant channel inflow

gaps, the flow rate is increasing as well, although $v_{\text {mean }}$ is decreasing. The occurring flow rates, relative to the ideal flow rate (without a gap), are shown in Fig. 16 on the right axis. As expected, larger gaps are leaking more, than smaller gaps. In practice, the effective flow rate will depend on the surface properties of the 3D-printed pumping case, the dimensions of the fluidic connectors, and certainly, the two anti-cyclically moving plugs, that are both leaking.

\subsubsection{Plug geometry}

The shape of the pumping plug is important for the resulting pumping pressure. To see the difference in the occurring pumping pressure, we simulated a fixed plug with constant gap to a tube channel. The differences with the simulations shown in Fig. 13 are the use of a static channel wall and a constant inflow velocity. The developing pressure in front of the plug is compared for varying plug geometries (Fig. 17).

A rectangular plug profile induces the highest pressure and, therefore, the best pump performance, but the applicability of the proposed device using external field sources favors the spherical plug.

\subsubsection{Transversal plug position}

The gap between plug and channel should be small enough for a good pump performance. We estimate the occurring flow rate with a centrally positioned plug (Fig. 18, 1). The simulation is performed with a blood-like fluid and a gap of $0.5 \mathrm{~mm}$

The realization of a control system to obtain a magnetically levitating plug in the center of the channel is a challenging task. It would require the use of complex equipment for sensing and actuation. Assisting trails on the channel surface would allow a central position of the sphere without excessive equipment (Fig. 18, 2), but since the dimension of such small parts (a few tenths of a millimeter) is very close to the minimum resolution of $3 \mathrm{D}$ prints, this solution is not practicable. In reality, the plug has a shifted position and contact to the inner wall (Fig. 18, 3). The sudden bouncing of the plug from one to the other side of the channel wall and the consequent occurring of turbulence and friction forces are not examined within this work.

To obtain the streamlines in the channels (Fig. 18), we use a fixed channel wall and a fixed plug. The fluid is flowing from left to right with a constant inflow velocity (same as in Section 3.2.5). For the computation of the flow rate in Fig. 19, we use a fixed plug and moving channel walls to imitate the plug movement (same as in Section 3.2.4). The flow rates occurring at a centered plug position are almost the same (with and without assisting trails), but a shifted plug position results in a performance loss. When looking at relative flow rates $Q / Q_{\text {ideal }}$ (again with the ideal flow rate $Q_{\text {ideal }}$ without a gap between plug and channel wall), assisting trails increase the pump performance, due to a smaller cross section area for the passing fluid.
Fig. 18 Static simulation of a spherical magnetic particle in fluid flow. The streamlines of passing fluid and the respective channel cross sections are shown with maximum velocity in red. A sphere is placed in a tube transversally (1) centered, (2) centered with assisting trails, and (3) shifted to one side of the wall

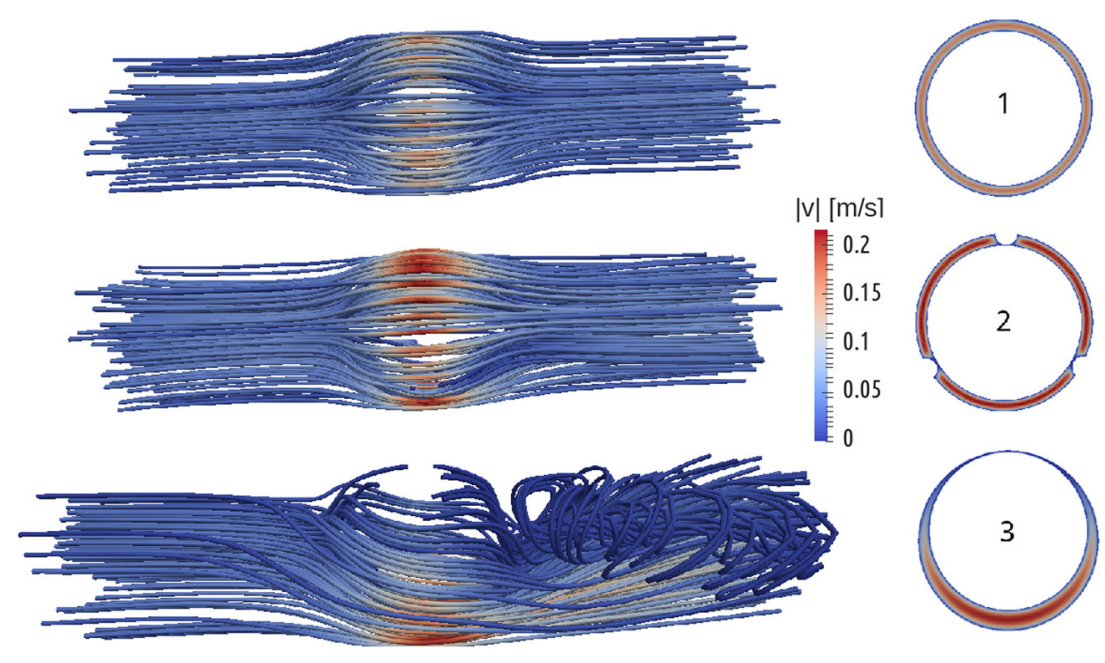




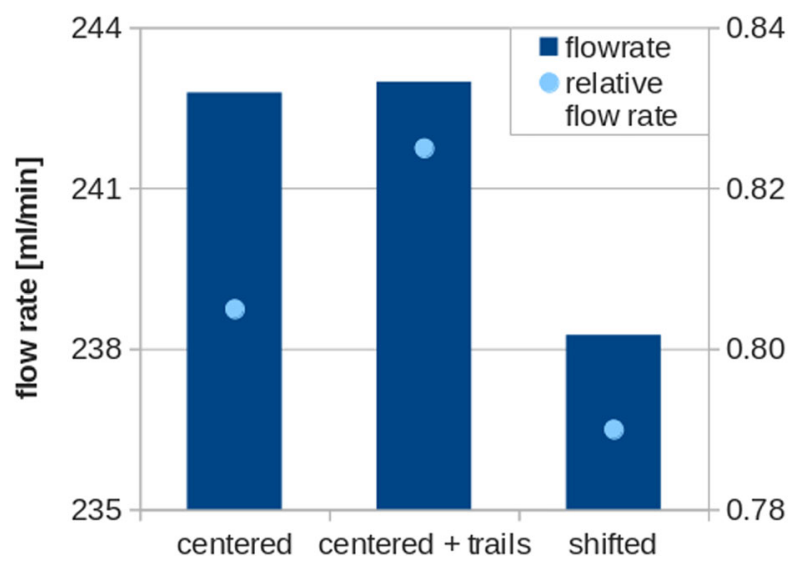

Fig. 19 The flow rates $Q$ in milliliters per minute and the relative flow rates $\left(Q / Q_{\text {ideal }}\right)$ are shown for different transversal positions of a spherical plug

\section{Experimental results}

\subsection{Biocompatibility}

As noted in Section 2.4.2, cytotoxicity tests are performed on materials if no information about their biocompatibility can be retrieved. The tubing and the 3D printing materials are not tested. The magnetic $\mathrm{NdFeB}$ plugs show to cause no impairment of cell viability (Fig. 20).

\subsection{Pump characterization}

The flow pattern delivered during the pumping cycles is shown in Fig. 21. During the active part of the cycle (A), the flow meter registers four peaks in the flow rate reflecting the four steps of the driving plug moving from position 9 to 3 (see also Fig. 1 on the right). Backflow is measured during the neutral part of the cycle $(\mathrm{N})$. The amount of backflow depends on the pressure afterload, since the system is open after the "valve" plug leaves position 6.

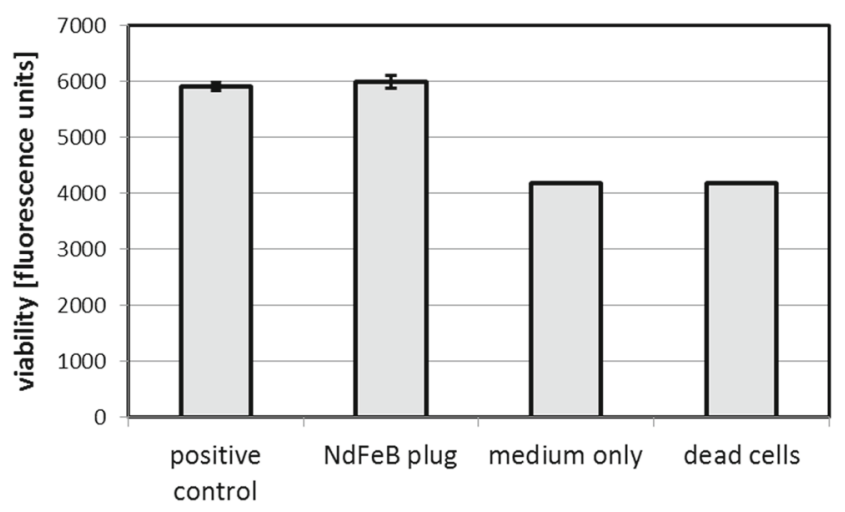

Fig. 20 Results of the cytotoxicity test on $\mathrm{NdFeB}$ plugs: the cells incubated in the $\mathrm{NdFeB}$ medium do not show any viability impairment in comparison to the normally cultured cells of the positive control

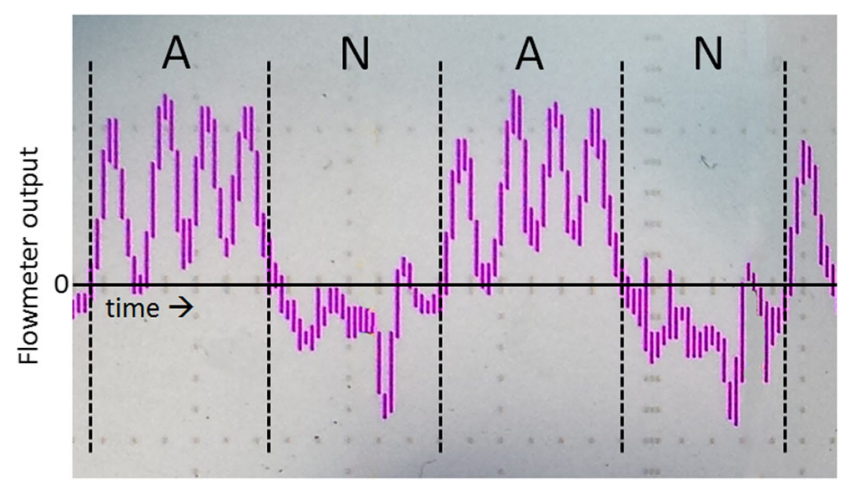

Fig. 21 Flow pattern of pumping cycle. Active (A) and neutral (N) parts of the cycle are highlighted

For the measurement of the pump performance, only the continuous component (average flow rate) delivered by the flow meter is taken into account.

The pump performance is experimentally measured for systems with a gap of 0.1 and $0.2 \mathrm{~mm}$ between plug and channel wall (more details in Section 2.4.1). In Fig. 22, the pressure load is plotted with the flow rate for water and a blood-like fluid. Duration times (dt) are set from 100 to $130 \mathrm{~ms}$, whereas the magnetomotive force is set such, that the system is running. For the viscous bloodlike fluid and a gap of $0.1 \mathrm{~mm}$, measurements are limited due to the missing performance of the electromagnetic actuation. The needed magnetomotive force is relatively high as seen in theory (Fig. 11). For a gap size of $0.2 \mathrm{~mm}$, we are using electromagnets with 100 windings. The applied current is about $4 \mathrm{~A}$, which gives us a magnetomotive force of about 400 AT. A stable pumping procedure can be achieved with the given duration times (dt). The magnetomotive force is higher, than theoretically needed; therefore, the plug is driven by a stop and go movement. A smaller gap of $0.1 \mathrm{~mm}$ increases the needed magnetomotive force drastically; the pump needs electromagnets with 300 windings and about $6 \mathrm{~A}$ of current (magnetomotive force of about 1800 AT). Due to the short activation of every single electromagnet, such high currents are possible, without massive overheating.

Pumping of water is possible as the theory predicts, yet pumping of blood-like fluid is difficult. The resulting pump performance of the blood-like fluid with a gap of $0.1 \mathrm{~mm}$ cannot be accurately measured. Here, we increase the currents even more and we have to untighten the plastic screws, that hold the two printed parts together (Fig. 2), by a delta of below $0.1 \mathrm{~mm}$ (resulting gap size between 0.1 and $0.2 \mathrm{~mm}$ ). The reduction of the gap between plug and channel from 0.2 to $0.1 \mathrm{~mm}$ approximately doubles the pump performance curves (comparing values at zero pressure and zero flow rate). The duration time has more influence on the pump performance when the gap is $0.2 \mathrm{~mm}$. 
Fig. 22 The performance curves relating delivered flow rate and afterload pressure are experimentally measured for water and blood-like fluid with a gap of 0.1 and $0.2 \mathrm{~mm}$. The problems of lacking magnetomotive force with a blood-like fluid and a gap of $0.1 \mathrm{~mm}$ are overcome with an increased current and loosened plastic screws (real gap size between 0.1 and $0.2 \mathrm{~mm}$ )
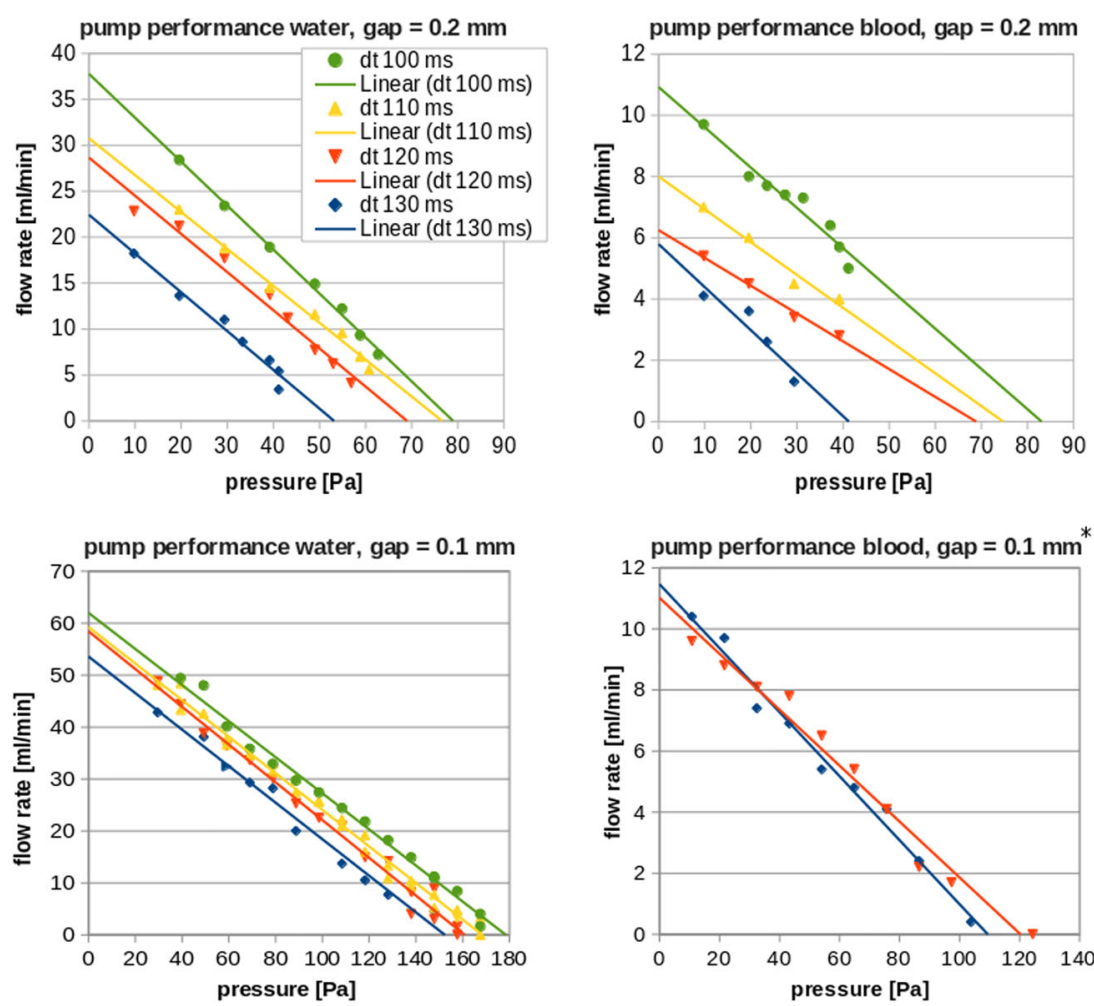

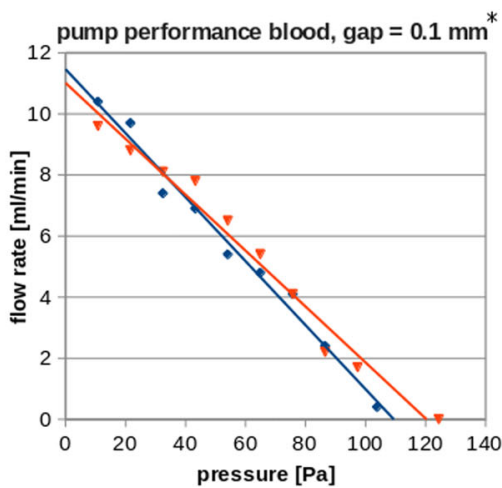

\section{Summary and conclusion}

In this paper, we show the development steps for a circular pumping system driven by anti-cyclical spherical permanent magnets. The theoretical model is solving the Newton's equation of motion in order to obtain the plugs velocities in the narrow tubing. The magnetomotive force of applied electromagnets and the design of the pump can be chosen such, that the plugs are subjected to a steady movement. Switching times and the distance between the electromagnets are essential for a proper functionality as well.

The theoretical model does not incorporate rotations of the plug, nor contacts with the channel wall. We believe that friction forces are important on the final performance, yet the theoretical values are in good agreement with the experimental results. Computer simulations of the fluid passing a fixed plug in a narrow tubing are providing possible flow rates for the final pump designs. Spherical plugs are preferred in the circular housing to reduce friction with the channel wall and to have a better plug control with electromagnets. An axially centered plug would optimally decrease friction and maximize the pump performance.

The computationally designed pump is fabricated by $3 \mathrm{D}$ printing. The flow rate of the pump is characterized experimentally. The comparison of the theoretical prediction with the experimental data suggests that increased pump performance will be possible by 3D printing technologies with higher resolution, so that surface roughness is reduced and the plug can be guided in the center by printed trails.

All materials used in the device are biocompatible. Cytotoxicity tests are performed on materials if no information about their biocompatibility can be retrieved from literature. The mechanical stimulation of particulate fluid, especially important in combination with blood, is not investigated within this work. The practical implementation of a solution to get axially centered plugs will have a major importance, not only on the pump performance but also on the application with cell-containing fluids. The damage of the cell membrane, whether caused by cell squeezing or high shear stress, is a critical issue. It will need to get investigated thoroughly by using for example a sensing platform for blood cell damage [18].

Acknowledgements Open access funding provided by Danube University Krems University for Continuing Education. The authors gratefully acknowledge the financial support of the NÖ Forschungs- und Bildungsges.m.b.H. (NFB) through the Life Science Calls (Project ID: LSC13-024).

Throughout the developing process, several 3D printing technologies of cooperation partners were used. The authors thank Markus Frauenschuh at Landesberufsschule Hallein, the research group of Dieter Suess at the Vienna University of Technology, and Bernd Bickel and Thomas Auzinger at the Institute of Science and Technology Austria for their personal assistance and hardware support. 
Open Access This article is distributed under the terms of the Creative Commons Attribution 4.0 International License (http:// creativecommons.org/licenses/by/4.0/), which permits unrestricted use, distribution, and reproduction in any medium, provided you give appropriate credit to the original author(s) and the source, provide a link to the Creative Commons license, and indicate if changes were made.

\section{References}

1. Laser DJ, Santiago JG (2004) A review of micropumps. J Micromech Microeng 14(6):R35

2. Yokota $S$ (2014) A review on micropumps from the viewpoint of volumetric power density. Mechanical Engineering Reviews 1(2):DSM0014-DSM0014

3. Hatch A, Kamholz AE, Holman G, Yager P, Bohringer KF (2001) A ferrofluidic magnetic micropump. J Microelectromech Syst 10(2):215-221

4. Al-Halhouli A, Kilani M, Büttgenbach S (2010) Development of a novel electromagnetic pump for biomedical applications. Sensors Actuators A Phys 162(2):172-176

5. Al Halhouli A, Kilani M, Waldschik A, Phataralaoha A, Büttgenbach S (2012) Development and testing of a synchronous micropump based on electroplated coils and microfabricated polymer magnets. Journal of Micromechanics and Microengineering 22(6):065,027

6. Giannatsis J, Dedoussis V (2009) Additive fabrication technologies applied to medicine and health care: a review. Int $\mathbf{J}$ Adv Manuf Technol 40(1):116-127

7. Vaezi M, Seitz H, Yang S (2013) A review on 3D microadditive manufacturing technologies. Int J Adv Manuf Technol 67(5-8):1721-1754
8. Gusenbauer M, Schrefl T (2018) Simulation of magnetic particles in microfluidic channels. J Magn Magn Mater 15(446):185-191

9. Xiong GM, Do AT, Wang JK, Yeoh CL, Yeo KS, Choong C (2015) Development of a miniaturized stimulation device for electrical stimulation of cells. J Biol Eng 9(1):14

10. Esch MB, Prot JM, Wang YI, Miller P, Llamas-Vidales JR, Naughton BA, Applegate DR, Shuler ML (2015) Multi-cellular 3D human primary liver cell culture elevates metabolic activity under fluidic flow. Lab Chip 15(10):2269-2277

11. Francis AW (1933) Wall effect in falling ball method for viscosity. J Appl Phys 4(11):403-406

12. Di Felice R (1996) A relationship for the wall effect on the settling velocity of a sphere at any flow regime. Int $\mathrm{J}$ Multiphase Flow 22(3):527-533

13. Gusenbauer M, Nguyen H, Reichel F, Exl L, Bance S, Fischbacher J, Özelt H, Kovacs A, Brandl M, Schrefl T (2014) Guided selfassembly of magnetic beads for biomedical applications. Phys B Condens Matter 435:21-24

14. Derby N, Olbert S (2010) Cylindrical magnets and ideal solenoids. Am J Phys 78(3):229-235

15. Gusenbauer M, Kovacs A, Reichel F, Exl L, Bance S, Özelt H, Schrefl T (2012) Self-organizing magnetic beads for biomedical applications. J Magn Magn Mater 324(6):977-982

16. Terfous A, Hazzab A, Ghenaim A (2013) Predicting the drag coefficient and settling velocity of spherical particles. Powder Technol 239:12-20

17. Lee $\mathrm{SH}, \mathrm{Wu} \mathrm{T}$ (2007) Drag force on a sphere moving in lowReynolds-number pipe flows. J Mech 23(04):423-432

18. Gusenbauer M, Mazza G, Brandl M, Schrefl T, Tóthová R, Jančigová I, Cimrák I (2016) Sensing platform for computational and experimental analysis of blood cell mechanical stress and activation in microfluidics. Procedia Eng 168:13901393 